\title{
COVID-19 outcomes in patients with familial Mediterranean fever: a retrospective cohort study
}

\author{
Serdar Can Güven ${ }^{1}$ (1) $\cdot$ Abdulsamet Erden $^{1} \cdot$ Özlem Karakaş $^{1} \cdot$ Berkan Armağan $^{1}$ - Eren Usul ${ }^{2} \cdot$ Ahmet Omma $^{1}$. \\ Orhan Küçükşahin ${ }^{3}$
}

Received: 3 January 2021 / Accepted: 9 February 2021 / Published online: 21 February 2021

(c) The Author(s), under exclusive licence to Springer-Verlag GmbH, DE part of Springer Nature 2021

\begin{abstract}
Aim of this study is to investigate the course of coronavirus disease 2019 (COVID-19), in our cohort of familial Mediterranean fever (FMF) patients in means of mortality, admission to hospital and/or intensive care unit and length of hospital stay.A retrospective cohort was formed from patients who have previously been followed with a diagnosis of FMF. Patients of this cohort were retrospectively evaluated for a positive severe acute respiratory syndrome-coronavirus 2 (SARS-CoV 2) polymerized chain reaction (PCR) test result and information regarding hospitalisation, intensive care unit admission and mortality were collected from medical records. Out of a total 496 FMF patients, 34 were detected to have a positive SARSCoV 2 PCR test. Eighty-five point three percent of these patients were under colchicine treatment and $17.6 \%$ were under interleukin (IL) - 1 inhibitor treatment. Eight of the 34 patients (23.9\%) were found to be hospitalized, one of them was admitted to the intensive care unit and died thereafter $(2.9 \%)$. An increasing trend in the frequency of comorbid diseases (presence of at least one comorbidity $64.7 \%$ in all patients vs $75.0 \%$ in hospitalized patients) and IL-1 inhibitor usage (17.6\% in all patients vs $50.0 \%$ in hospitalized patients) was observed in hospitalized patients.Rates of comorbid diseases and IL-1 inhibitor use for FMF were observed to be increased in FMF patients hospitalized for COVID-19.
\end{abstract}

Keywords Familial $\cdot$ Mediterranean $\cdot$ Fever $\cdot$ Coronavirus $\cdot$ Outcome

$\begin{array}{ll}\text { Abbreviations } \\ \text { COVID-19 } & \text { Coronavirus disease 2019 } \\ \text { FMF } & \text { Familial Mediterranean fever } \\ \text { ICU } & \text { Intensive care unit } \\ \text { IL-1 } & \text { Interleukin 1 } \\ \text { MEDULA } & \begin{array}{l}\text { Social Security Institution Medical } \\ \text { Messenger }\end{array} \\ \text { MEFV } & \text { Mediterranean fever gene } \\ \text { PCR } & \text { Polymerized chain reaction } \\ \text { SARS-CoV 2 } & \text { Severe acute respiratory syndrome-coro- } \\ & \text { navirus 2 }\end{array}$

Serdar Can Güven

drserdarguven@gmail.com

1 Clinic of Rheumatology, Ministry of Health Ankara City Hospital, Bilkent, Çankaya, Ankara, Turkey

2 Clinic of Emergency Medicine, Sincan Dr. Nafiz Körez State Hospital, Ankara, Turkey

3 Department of Internal Medicine, Division of Rheumatology, Ankara Yıldırım Bayezıt University Medical School, Ankara, Turkey
SPSS

TNF $\alpha$

Statistical Package for the Social Sciences

Tumor necrosis factor alpha

\section{Introduction}

Coronavirus disease 2019 (COVID-19) pandemic, caused by severe acute respiratory syndrome-coronavirus 2 (SARS-CoV 2), has become a major global health issue since December 2019. The disease course is heterogeneous. Although most patients suffer mildly, a significant portion of the patients develops fatal complications such as acute respiratory distress syndrome, multiorgan failure and a hyperimmune state so-called "cytokine storm", which is characterized by the extreme release of various cytokines and chemokines, disequilibrium in distribution of T-cell subsets and related with a poor prognosis [1-3]. The true cause of this state is yet to be fully clarified but hypothetically a hyperreactive immune system, prolonged inflammatory response due to delayed viral clearance or immune dysregulation may be the underlying reasons [3]. 
Activation of inflammasomes by viral proteins and release of proinflammatory cytokines is a natural response of innate immunity to viral infections however, a dysregulated inflammasome activity may also result in an aberrant inflammatory response. Likewise, in Familial Mediterranean fever (FMF), a hereditary innate immunity disorder due to gain of function mutations in Mediterranean fever gene (MEFV) encoding pyrin protein, a hyperactive pyrin inflammasome leads to febrile inflammatory episodes sharing similarities with COVID-19-associated cytokine storm [4, 5]. As mentioned before, an already hyperresponsive innate immune system may increase susceptibility developing cytokine storm and severe symptoms in COVID-19. On the other hand, the presence of $M E F V$ mutations were suggested to be advantageous against some infections [6]. Therefore it is intriguing whether the hyperactive innate immunity of FMF patients would provide protection against COVID-19 or lead to a more severe disease course.

Colchicine and interleukin 1 (IL-1) antagonists, the mainstay of treatment in FMF with well-established effectiveness in controlling hyperinflammation, have also been investigated in the management of COVID-19 and promising results have been observed [7-16]. It is also a matter of interest whether in FMF patients who already receive these agents, a preventive effect against the development of cytokine storm during COVID-19 would be observed.

There is scarce knowledge in the literature regarding COVID-19 course in autoinflammatory conditions like FMF, particularly in adult patients [6,16-19]. To further contribute to the knowledge in this subject, we aimed in our study to evaluate outcomes of COVID-19 in our cohort of FMF patients in means of mortality, admission to hospital and/or intensive care unit (ICU) and length of hospital stay.

\section{Method}

\section{Study design}

This study was designed as a cross-sectional, retrospective cohort study with approval by Ankara City Hospital ethics committee and was therefore performed in accordance with the ethical standards laid down in the 1964 Declaration of Helsinki and its later amendments. An official permission was obtained from the Ministry of Health, to conduct this study dated 21 Jul 2020.

\section{Patients}

A retrospective cohort was formed from FMF patients meeting Tel-Hashomer criteria [20], who have previously been followed by authors (AE, AO, BA, OK, SCG) in either Ankara City Hospital or Ankara Numune Training and Research Hospital or Ankara Atatürk Training and Research Hospital (the latter two centers were merged to found Ankara City Hospital in 2019 and have been working as a single-center since clinical records of all three centers are accessible via Ankara City Hospital database). Between December 1 and 15, 2020, patients of this cohort were retrospectively investigated for a SARS-CoV 2 polymerized chain reaction (PCR) test result from Public Health Management System (HSYS), to which all cases with a PCR test were registered during the pandemic in our country. Patients older than 18 years of age with a recorded positive PCR test result from nasopharyngeal swab between March 11 and December 1, 2020 were enrolled in the study.

\section{Main outcomes and other variables}

Rates of hospitalization, ICU admission and mortality due to COVID-19 were selected as main outcome variables and collected from medical records and HSYS. Data regarding to demographics, comorbidities, $M E F V$ mutations and medical treatment administered for FMF were collected from the Ankara City hospital database. Adherence to medical treatments were confirmed by the presence of consecutive drug prescriptions due date of positive SARS-CoV 2 PCR test in Social Security Institution Medical Messenger (MEDULA) records since the pandemic of COVID-19 interrupted regular follow-ups.

\section{Statistical analysis}

Data were analyzed using Statistical Package for the Social Sciences (SPSS) 22.0 software. Normality of continuous variables was evaluated with Shapiro-Wilk test and with plots and histograms visually. Continuous variables were presented either with median (minimum-maximum) or mean \pm standard deviation, according to normality. Categorical variables are presented with numbers and percentages.

\section{Results}

\section{Clinical features}

Our cohort included a total of 496 FMF patients. Among these, 34 patients were detected to have a positive SARSCoV 2 PCR test. Demographics, comorbid diseases and drugs for active FMF treatment of these patients were given in Table 1. Median age was 40.5 years and $64.7 \%$ of the patients had at least one comorbid disease, hypertension being the most frequent (23.5\%). Eighty-five point three percent of these patients were under colchicine treatment and $17.6 \%$ were under IL- 1 inhibitor treatment (all of which was anakinra). Three of the patients (8.8\%) were detected to have 
Table 1 Demographics, comorbidities, active familial Mediterranean fever medications and coronavirus disease 2019 outcomes in familial Mediterranean fever patients with a positive severe acute respiratory syndromecoronavirus 2 polymerized chain reaction test

\begin{tabular}{|c|c|c|}
\hline & All $(n=34)$ & Hospitalized $(n=8)$ \\
\hline Age, years, median (min-max) & $40.5(23-59)$ & $43.5(38-59)$ \\
\hline Gender, male, number $(\%)$ & $20(58.8)$ & $3(37.5)$ \\
\hline Patients with $\geq 1$ comorbidities, number (\%) & $22(64.7)$ & $6(75)$ \\
\hline Patients with $\geq 2$ comorbidities, number $(\%)$ & $8(23.5)$ & $4(50)$ \\
\hline \multicolumn{3}{|l|}{ Comorbidities, number (\%) } \\
\hline Hypertension & $8(23.5)$ & $4(50)$ \\
\hline Diabetes & $3(8.8)$ & $3(37.5)$ \\
\hline Hyperlipidemia & $1(2.9)$ & $0(0)$ \\
\hline Asthma & $3(8.8)$ & $1(12.5)$ \\
\hline Hypothyroidism & $3(8.8)$ & $2(25)$ \\
\hline Coronary artery disease & $2(5.8)$ & $1(12.5)$ \\
\hline Congestive heart failure & $1(2.9)$ & $1(12.5)$ \\
\hline End-stage renal disease & $1(2.9)$ & $0(0)$ \\
\hline Spondyloarthritis & $6(17.6)$ & $2(25)$ \\
\hline Proteinuria $>1 \mathrm{~g} /$ day & $3(8.8)$ & $2(25)$ \\
\hline Others & $7(20.5)$ & $1(12.5)$ \\
\hline \multicolumn{3}{|l|}{$\begin{array}{l}\text { Active medical treatment for familial Mediterranean fever, } \\
\text { number }(\%)\end{array}$} \\
\hline Colchicine & $29(85.3)$ & $8(100)$ \\
\hline IL-1 inhibitors & $6(17.6)$ & $4(50)$ \\
\hline TNF $\alpha$ inhibitors & $1(2.9)$ & $0(0)$ \\
\hline \multicolumn{3}{|l|}{ Coronavirus disease 2019 outcomes } \\
\hline Hospitalization, number (\%) & $8(23.5)$ & \\
\hline Length of hospital stay (days), median (min-max) & $7.5(3-19)$ & \\
\hline ICU admission, number (\%) & $1(2.9)$ & \\
\hline Death, number $(\%)$ & $1(2.9)$ & \\
\hline
\end{tabular}

$I L-1$ interleukin-1, TNF $\alpha$ tumor necrosis factor alpha, $I C U$ intensive care unit proteinuria exceeding $1 \mathrm{~g}$ per day (Table 1 ). Mediterranean fever gene $(M E F V)$ mutations were obtained from hospital records in 14 out of 34 patients (five $M 694 \mathrm{~V}$ homozygous, four $M 694 \mathrm{~V}$ heterozygous, two $V 726 \mathrm{~A}$ heterozygous, one M680I homozygous, one M680I heterozygous, one E167D/ F479L compound heterozygous).

\section{Outcomes}

Eight of the 34 patients (23.5\%) were found to be hospitalized for COVID-19 and one of them was admitted to ICU and died thereafter (Table 1). This patient was female, aged 58 years and had multiple comorbidities including diabetes mellitus, hypertension, congestive heart failure and proteinuria $>1 \mathrm{gr} /$ day. She was both under colchicine and anakinra treatments. None of the other patients were admitted to ICU or died. Median length of hospital stay was found to be 7.5 days. Fifty percent of the hospitalized patients had at least two comorbid diseases. An increasing trend in the frequency of comorbid diseases (presence of at least one comorbidity: $64.7 \%$ in all patients vs $75.0 \%$ in hospitalized patients) and IL-1 inhibitor usage (17.6\% in all patients vs
$50.0 \%$ in hospitalized patients) was observed in hospitalized patients (Table 1). Two out of three patients with proteinuria were hospitalized.

\section{Discussion}

Our results demonstrated that among 34 FMF patients with a positive SARS-CoV 2 PCR test, eight of them were hospitalized and one admitted to ICU and died consequently. The lost patient was relatively older, had multiple comorbidities including proteinuria and was under IL-1 inhibitor treatment. Comorbid disease burden and administration of IL-1 blockers for FMF treatment was observed to be increased in hospitalized patients.

Age, male gender, body mass index, presence of comorbid diseases and certain antirheumatic drugs (rituximab, mycophenolate mophetil, steroids) were reported to be related to worse outcomes in rheumatic conditions [19,21]. However, there are limited knowledge about COVID-19 outcomes in FMF patients [16, 18]. Course of COVID-19 in FMF patients is a matter of interest since FMF attacks 
share similarities with COVID-19 related cytokine storm [3]. It can be speculated that the already hyperreactive innate immunity of FMF patients with defective inflammasomes, may increase susceptibility to hyperinflammatory response in COVID-19. In one study including a relatively large number of FMF patients, Bourguiba et al. [17], reported seven hospitalizations and two deaths out of 27 patients infected with SARS-CoV 2 in a cohort of 342 FMF patients, suggesting a similar disease course with the general population. Likewise, in a large study from France in which COVID19 survival was evaluated in various rheumatic conditions, two deaths out of 15 periodic fever patients were reported without significantly increased risk of death when compared to other rheumatic conditions. However, the authors suggested cautious interpretation of the results for autoinflammatory diseases due to a very low number of patients [19]. We observed $23.9 \%$ hospitalization and $2.9 \%$ mortality rate in 34 patients in our study.

Mainstay treatment agents of FMF, colchicine and IL-1 inhibitors particularly anakinra, have also been considered in the treatment of COVID-19. There are several reports of anakinra administration in severe COVID-19 with promising results [11-15]. However, among our FMF patients with COVID-19, four out of six anakinra users were hospitalized and one of them eventually died. Likewise, Bourguiba et al. [17] reported two deaths in four anakinra users. Since anakinra is generally used in FMF patients with amyloidosis or colchicine resistance, anakinra administration may indicate a rather severe disease course in FMF patients. Therefore, it is unclear whether this trend is due to long-term IL-1 inhibition or to disease burden.

Two out of three patients with urine protein excretion over $1 \mathrm{~g}$ per day were hospitalized in our study. Although such excessive proteinuria is highly suggestive of concomitant amyloidosis in FMF, none of these patients had a histopathologic confirmation therefore direct assumptions should be avoided. Regardless, the presence of severe proteinuria may be an important contributor to worse prognosis in FMF patients with COVID-19. Infections have previously been reported as the leading cause of mortality in AA amyloidosis and presence of nephrotic syndrome was discussed as a potential contributor to overall immunosuppression in these patients [22]. However, our patient population is insufficient to prove such deduction.

Retrospective nature of the study, small sample size and lack of control group indicate cautious interpretation of our results. The absence of clinical diagnosis of COVID-19 and biopsy-proven amyloidosis are other limitations of our study. In addition, the effects of chronic colchicine use on COVID-19 course cannot be interpreted with our results. Furthermore, some COVID-19 cases in our cohort may have been missed due to asymptomatic or mild disease course. Nevertheless, we think our results are still valuable since there is scarce data in the literature regarding COVID-19 outcomes in FMF patients.

\section{Conclusions}

Our results demonstrated mortality and hospitalization rates in FMF patients with COVID-19 similar to the previous reports in the literature. Frequency of comorbid diseases, proteinuria and IL-1 inhibitor use for FMF were tended to be higher in hospitalized patients. Proteinuria and IL-1 inhibitor use may be related to the disease severity of FMF. However, larger studies are needed for more accurate conclusions about COVID-19 outcomes in FMF patients.

Acknowledgements Authors declare none.

Author contributions All authors substantially contributed to study design and acquisition, analysis and interpretation of the data. All authors revised the final version of the work and approved it for publication in agreement on all aspects of the study. There is no editing support to acknowledge.

Funding This research did not receive any grant.

Data availability The datasets used in this study are available from the corresponding author on valid request.

\section{Compliance with ethical standards}

Conflict of interest The authors declare that they have no conflicts of interest.

Ethics approval The study protocol was approved by the institutional committee on Human Research Ethics.

\section{References}

1. Mehta P, McAuley DF, Brown M et al (2020) COVID-19: consider cytokine storm syndromes and immunosuppression. Lancet 395:1033-1034. https://doi.org/10.1016/S0140-6736(20)30628-0

2. Tufan A, Avanoğlu Güler A, Matucci-Cerinic M (2020) COVID19 , immune system response, hyperinflammation and repurposing antirheumatic drugs. Turk J Med Sci 50:620-632. https://doi. org/10.3906/sag-2004-168

3. Fajgenbaum DC, June CH (2020) Cytokine storm. N Engl J Med 383:2255-2273. https://doi.org/10.1056/NEJMra2026131

4. Stella A, Lamkanfi M, Portincasa P (2020) Familial mediterranean fever and COVID-19: friends or foes? Front Immunol 18(11):574593. https://doi.org/10.3389/fimmu.2020.574593

5. Tufan A, Lachmann HJ (2020) Familial Mediterranean fever, from pathogenesis to treatment: a contemporary review. Turk J Med Sci 50:1591-1610. https://doi.org/10.3906/sag-2008-11

6. Batu ED, Özen S (2020) Implications of COVID-19 in pediatric rheumatology. Rheumatol Int 40:1193-1213. https://doi. org/10.1007/s00296-020-04612-6

7. Sandhu T, Tieng A, Chilimuri S et al (2020) A case control study to evaluate the impact of colchicine on patients admitted 
to the hospital with moderate to severe COVID-19 infection. Can J Infect Dis Med Microbiol 2020:8865954. https://doi. org/10.1155/2020/8865954

8. Brunetti L, Diawara O, Tsai A et al (2020) Colchicine to weather the cytokine storm in hospitalized patients with COVID-19. J Clin Med 9:2961. https://doi.org/10.3390/jcm9092961

9. Scarsi M, Piantoni S, Colombo E et al (2020) Association between treatment with colchicine and improved survival in a single-centre cohort of adult hospitalised patients with COVID-19 pneumonia and acute respiratory distress syndrome. Ann Rheum Dis 79:1286-1289. https://doi.org/10.1136/annrheumdis-2020-21771 2

10. Deftereos SG, Giannopoulos G, Vrachatis DA et al (2020) Effect of colchicine vs standard care on cardiac and inflammatory biomarkers and clinical outcomes in patients hospitalized with coronavirus disease 2019: the GRECCO-19 randomized clinical trial. JAMA Netw Open 3:e2013136. https://doi.org/10.1001/jaman etworkopen.2020.13136

11. Iglesias-Julián E, López-Veloso $\mathrm{M}$, de-la Torre-Ferrera $\mathrm{N}$ et al (2020) High dose subcutaneous Anakinra to treat acute respiratory distress syndrome secondary to cytokine storm syndrome among severely Ill COVID-19 patients. J Autoimmun 115:102537. https ://doi.org/10.1016/j.jaut.2020.102537

12. Huet $\mathrm{T}$, Beaussier $\mathrm{H}$, Voisin $\mathrm{O}$ et al (2020) Anakinra for severe forms of COVID-19: a cohort study. Lancet Rheumatol 2:e393e400. https://doi.org/10.1016/S2665-9913(20)30164-8

13. Kooistra EJ, Waalders NJB, Grondman I et al (2020) Anakinra treatment in critically ill COVID-19 patients: a prospective cohort study. Crit Care 24:688. https://doi.org/10.1186/s13054-02003364-w

14. Balkhair A, Al-Zakwani I, Al Busaidi M et al (2020) Anakinra in hospitalized patients with severe COVID-19 pneumonia requiring oxygen therapy: results of a prospective, open-label, interventional study. Int J Infect Dis 103:288-296. https://doi.org/10.1016/j. ijid.2020.11.149

15. Erden A, Ozdemir B, Karakas O et al (2020) Evaluation of 17 patients with COVID-19 pneumonia treated with anakinra according to HScore, SOFA, MuLBSTA, and Brescia-COVID respiratory severity scale (BCRSS) scoring systems. J Med Virol. https://doi.org/10.1002/jmv.26473

16. Haslak F, Yildiz M, Adrovic A et al (2020) Management of childhood-onset autoinflammatory diseases during the COVID-19 pandemic. Rheumatol Int 40:1423-1431. https://doi.org/10.1007/ s00296-020-04645-x

17. Bourguiba R, Delplanque M, Vinit C et al (2020) Clinical course of COVID-19 in a cohort of 342 familial Mediterranean fever patients with a long-term treatment by colchicine in a French endemic area. Ann Rheum Dis. https://doi.org/10.1136/annrh eumdis-2020-218707

18. Meyts I, Bucciol G, Quinti I et al (2020) Coronavirus disease 2019 in patients with inborn errors of immunity: an international study. J Allergy Clin Immunol. https://doi.org/10.1016/j. jaci.2020.09.010

19. FAI2R /SFR/SNFMI/SOFREMIP/CRI/IMIDIATE consortium and contributors (2020) Severity of COVID-19 and survival in patients with rheumatic and inflammatory diseases: data from the French RMD COVID-19 cohort of 694 patients. Ann Rheum Dis. https://doi.org/10.1136/annrheumdis-2020-218310

20. Sohar E, Gafni J, Pras M, Heller H (1967) Familial Mediterranean fever. A survey of 470 cases and review of the literature. Am J Med 43:227-253

21. Hyrich KL, Machado PM (2020) Rheumatic disease and COVID19: epidemiology and outcomes. Nat Rev Rheumatol. https://doi. org/10.1038/s41584-020-00562-2

22. Terré A, Deshayes S, Savey L et al (2020) Cause of death and risk factors for mortality in AA amyloidosis: a French retrospective study. Eur J Intern Med 82:130-132. https://doi.org/10.1016/j. ejim.2020.08.012

Publisher's Note Springer Nature remains neutral with regard to jurisdictional claims in published maps and institutional affiliations. 